\title{
Biomarker progressions explain higher variability in stage-specific cognitive decline than baseline values in Alzheimer disease
}

\author{
Hiroko H. Dodge ${ }^{\mathrm{a}, \mathrm{b}, \mathrm{c}, *}$, Jian Zhu ${ }^{\mathrm{d}}$, Danielle Harvey ${ }^{\mathrm{e}}$, Naomi Saito ${ }^{\mathrm{e}}$, Lisa C. Silbert ${ }^{\mathrm{a}, \mathrm{f}}$, \\ Jeffrey A. Kaye ${ }^{\mathrm{a}, \mathrm{f}}$, Robert A. Koeppe ${ }^{\mathrm{g}}$, Roger L. Albin ${ }^{\mathrm{b}, \mathrm{c}, \mathrm{h}}$, for the Alzheimer's Disease \\ Neuroimaging Initiative ${ }^{1}$ \\ ${ }^{a}$ Department of Neurology, Layton Aging and Alzheimer's Disease Center, Oregon Health \& Science University, Portland, OR \\ ${ }^{b}$ Department of Neurology, University of Michigan, Ann Arbor, MI \\ ${ }^{c}$ Michigan Alzheimer's Disease Center, University of Michigan, Ann Arbor, MI \\ ${ }^{d}$ Department of Biostatistics, University of Michigan, Ann Arbor, MI \\ ${ }^{e}$ Department of Public Health Sciences, University of California, Davis, CA \\ ${ }^{f}$ Portland Veteran Affairs Medical Center, Portland, OR \\ ${ }^{g}$ Department of Radiology, University of Michigan, Ann Arbor, MI \\ ${ }^{h}$ Neurology Service and Geriatric Research, Education and Clinical Center, Veterans Affairs Ann Arbor Healthcare System, Ann Arbor, MI
}

\begin{abstract}
Background: It is unknown which commonly used Alzheimer disease (AD) biomarker valuesbaseline or progression-best predict longitudinal cognitive decline.

Methods: 526 subjects from the Alzheimer's Disease Neuroimaging Initiative (ADNI). ADNI composite memory and executive scores were the primary outcomes. Individual-specific slope of the longitudinal trajectory of each biomarker was first estimated. These estimates and observed baseline biomarker values were used as predictors of cognitive declines. Variability in cognitive declines explained by baseline biomarker values was compared with variability explained by biomarker progression values.

Results: About $40 \%$ of variability in memory and executive function declines was explained by ventricular volume progression among mild cognitive impairment patients. A total of $84 \%$ of memory and $65 \%$ of executive function declines were explained by fluorodeoxyglucose positron emission tomography (FDG-PET) score progression and ventricular volume progression, respectively, among AD patients.

Conclusions: For most biomarkers, biomarker progressions explained higher variability in cognitive decline than biomarker baseline values. This has important implications for clinical trials targeted to modify AD biomarkers.
\end{abstract}

(C) 2014 The Alzheimer's Association. All rights reserved.

Keywords: $\quad$ ADNI; Cognitive declines; Biomarker; Biomarker progressions; ADNI-mem; ADNI-exe; MCI; FDG-PET; MRI volume

\footnotetext{
${ }^{1}$ Data used in preparation of this article were obtained from the Alzheimer's Disease Neuroimaging Initiative (ADNI) database (adni.loni.usc. edu). As such, the investigators within the ADNI contributed to the design and implementation of ADNI and/or provided data but did not participate in analysis or writing of this report. A complete listing of ADNI investigators can be found at: http://adni.loni.ucla.edu/wp-content/uploads/how_to_ apply/ADNI_Acknowledgement_List.pdf.

*Corresponding author. Tel.: 503-494-6977; Fax: 503-494-7499.

E-mail address: dodgeh@ohsu.edu
}

\section{Introduction}

The cascade model of Alzheimer's disease (AD) pathologic progression hypothesizes a specific sequence of pathologic events involving the formation of amyloidbased neuritic plaques, now accepted to occur many years before symptomatic onset, followed by tau-based neurofibrillary pathology, changes in brain structure and function, and finally cognitive impairment and functional disability. This model remains hypothetical with the timing 
Table 1

Baseline characteristics of samples (from ADNI 1)

\begin{tabular}{|c|c|c|c|c|c|c|c|c|c|}
\hline & \multicolumn{3}{|c|}{ Normal at baseline } & \multicolumn{3}{|c|}{ MCI at baseline } & \multicolumn{3}{|c|}{$\mathrm{AD}$ at baseline } \\
\hline Age & 156 & N/A & $75.0(4.8)$ & 262 & N/A & $74.2(7.4)$ & 108 & N/A & $74.0(7.7)$ \\
\hline $\begin{array}{l}\text { Apoe } 4 \text { (e4 allele } \\
\text { present) }(\%)\end{array}$ & 156 & N/A & 28.2 & 262 & N/A & 55.3 & 108 & N/A & 67.6 \\
\hline CSF t-tau $(\mathrm{pg} / \mathrm{mL})$ & 79 & $1.8(1-2)$ & $69.4(30.1)$ & 131 & $1.7(1-2)$ & $104.1(51.9)$ & 61 & $1.5(1-2)$ & $120.3(48)$ \\
\hline CSF A $\beta 42(\mathrm{pg} / \mathrm{mL})$ & 79 & $1.8(1-2)$ & $206.4(50.5)$ & 131 & $1.7(1-2)$ & $168(58.5)$ & 61 & $1.5(1-2)$ & $142.4(38.3)$ \\
\hline FDG-PET & 71 & $3.8(1-5)$ & $1.3(0.1)$ & 136 & $4.1(1-6)$ & $1.2(0.1)$ & 53 & $2.8(1-4)$ & $1.1(0.1)$ \\
\hline \multicolumn{10}{|l|}{ Brain volume $\left(\mathrm{cm}^{3}\right)$} \\
\hline WMH & 156 & $3.4(1-5)$ & $7.4 \mathrm{E}-4(2 \mathrm{E}-3)$ & 262 & $3.6(1-5)$ & $8.5 \mathrm{E}-4(3 \mathrm{E}-3)$ & 107 & $2.5(1-3)$ & $1.1 \mathrm{E}-3(3 \mathrm{E}-3)$ \\
\hline Hippocampal/ICV & 156 & $4.2(1-5)$ & $0.2 \%(0.03 \%)$ & 262 & $4.3(1-6)$ & $0.2 \%(0.03 \%)$ & 108 & $2.9(1-4)$ & $0.2 \%(0.03 \%)$ \\
\hline Ventricular/ICV & 155 & $4.2(1-5)$ & $1.1 \%(0.5 \%)$ & 262 & $4.3(1-6)$ & $1.3 \%(0.6 \%)$ & 108 & $2.9(1-4)$ & $1.5 \%(0.7 \%)$ \\
\hline Total brain/ICV & 156 & $4.2(1-5)$ & $68.8 \%(4.0 \%)$ & 262 & $4.3(1-6)$ & $66.7 \%(4.2 \%)$ & 108 & $2.9(1-4)$ & $65.1 \%(4.2 \%)$ \\
\hline \multicolumn{10}{|l|}{ Thickness (mm) } \\
\hline Precuneus thickness & 156 & $4.2(1-5)$ & $2.1(0.2)$ & 262 & $4.3(1-6)$ & $2.0(0.2)$ & 108 & $2.9(1-4)$ & $1.95(0.2)$ \\
\hline $\begin{array}{l}\text { Medial temporal } \\
\text { thickness }{ }^{\dagger}\end{array}$ & 156 & $4.2(1-5)$ & $6.0(0.5)$ & 262 & $4.3(1-6)$ & $5.4(0.8)$ & 108 & $2.9(1-4)$ & $4.9(0.7)$ \\
\hline
\end{tabular}

Abbreviations: CSF, cerebrospinal fluid; FDG-PET, fluorodeoxyglucose positron emission tomography; MCI, mild cognitive impairment; WMH, white matter hyperintensity; ICV, intracranial volume.

$* \mathrm{~N}$ at baseline.

${ }^{\dagger}$ Summary variable by adding averaged means for left and right entorhinal, perirhinal, and posterior parahipplocampal cortical region thickness.

of each stage in relation to disease progression yet to be confirmed. Accumulated data, however, support this model and it provides a useful framework for investigating the properties of different biomarkers [1,2]. Clinical trials would be improved by identifying the biomarkers most strongly associated with cognitive and functional declines at each stage of $\mathrm{AD}$. Identifying biomarkers associated with subtle declines in cognitive functions among cognitively normal and mildly affected subjects is especially critical as research efforts move toward early identification of high risk subjects and prevention of progression.

One issue not examined systematically across various biomarkers is which component of commonly used AD biomarkers-baseline value or progression of biomarker values (biomarker progressions) - is more strongly associated with cognitive declines. In the cascade model $[1,2]$, the capacity of each biomarker to predict cognitive decline depends on the stage of $\mathrm{AD}$ disease process (e.g., normal, early mild cognitive impairment [MCI], late $\mathrm{MCI}$, or $\mathrm{AD}$ ), and whether biomarker baseline values or biomarker progressions are used. It is likely, for example, that brain beta amyloid burden is already high and probably plateaus by the time of $\mathrm{AD}$ diagnosis $[3,4]$, and although brain amyloid burden may distinguish among subjects with $\mathrm{AD}, \mathrm{MCI}$, and normal subjects cross-sectionally, continuing declines in cognitive functions at late MCI or AD stages will not be related to brain amyloid burden. Although baseline biomarker values are examined often in relation with subsequent longitudinal cognitive or functional trajectories, there is a paucity of data regarding biomarker progressions and their associations with cognitive or functional trajectories. Examining the relative ability of baseline values versus biomarker progressions at each stage of $\mathrm{AD}$ in explaining cognitive trajectories could improve clinical trial designs by allowing the recruitment of high risk populations with higher accuracy. We used data from the Alzheimer's Disease Neuroimaging Initiative study (ADNI-1) to examine which components (baseline values or biomarker progressions) are associated with declines in memory and executive cognitive functions. To conduct a fair comparison across different biomarkers and to increase clinical applicability of our results, we standardized all biomarkers and provided the clinical values corresponding to each standard deviation.

\section{Methods}

\subsection{Data source}

Data used in the preparation of this article were obtained from the ADNI database (adni.loni.usc.edu). The ADNI was launched in 2003 by the National Institute on Aging, the National Institute of Biomedical Imaging and Bioengineering, the Food and Drug Administration, private pharmaceutical companies, and nonprofit organizations as a $\$ 60$ million, 5-year public-private partnership. The primary goal of 

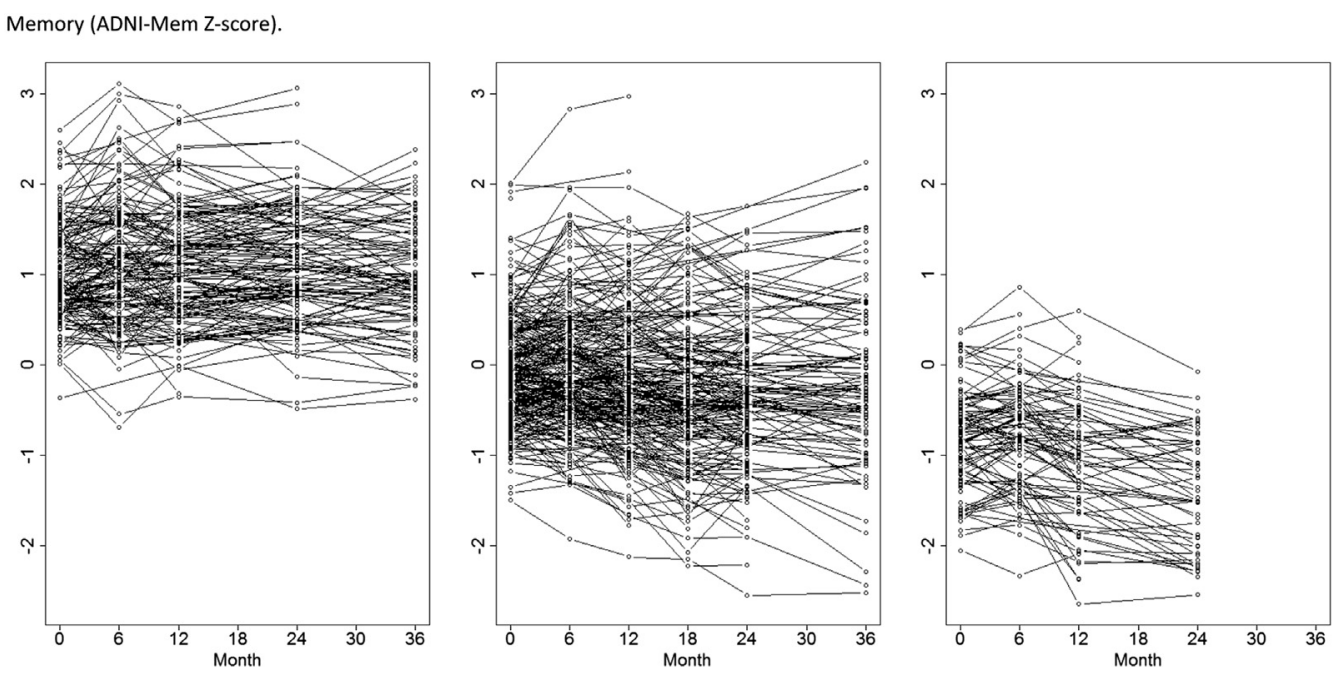

Executive function (ADNI-Exe Z-score)
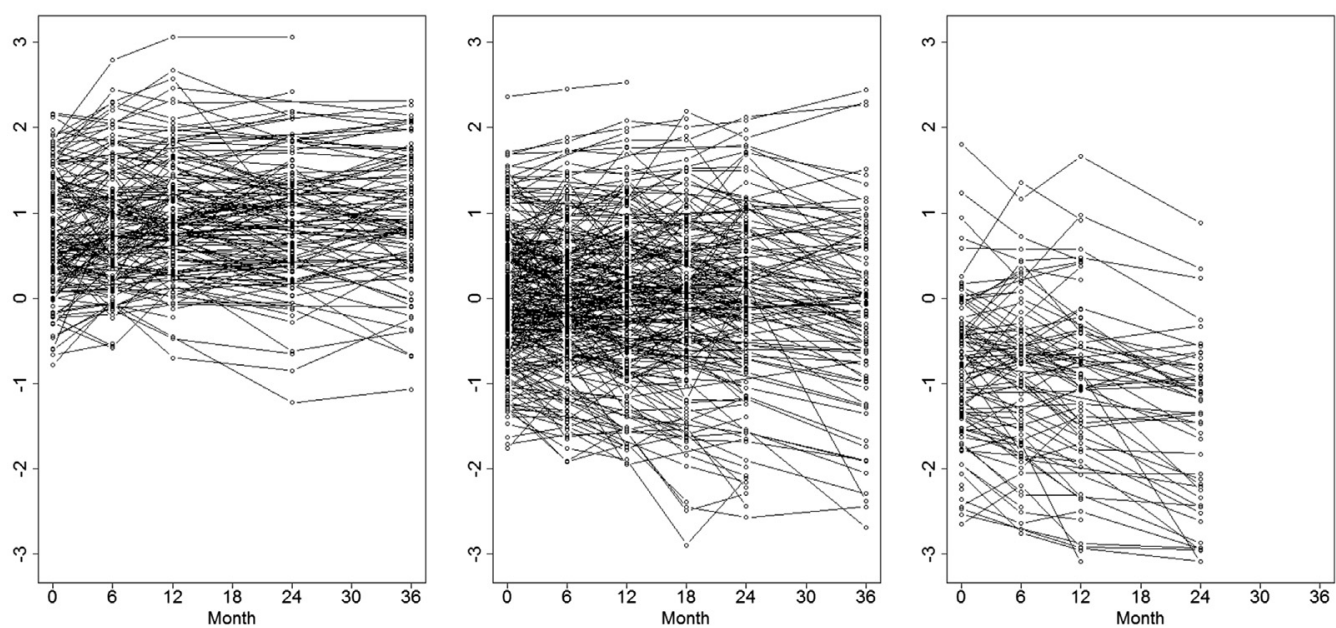

Fig. 1. Spaghetti plots of cognitive functions and biomarkers used in the analysis among subjects diagnosed as normal at baseline (left figures), diagnosed as mild cognitive impairment (MCI) at baseline (middle figures), and diagnosed as Alzheimer's disease (AD) at baseline (right figures).

ADNI is to test whether serial magnetic resonance imaging (MRI), positron emission tomography (PET), other biological markers, and clinical and neuropsychological (NP) assessment can be combined to measure the progression of MCI and early $\mathrm{AD}$. The determination of sensitive and specific markers of very early AD progression is intended to aid researchers and clinicians to develop new treatments and monitor their effectiveness, and lessen the time and cost of clinical trials. The principal investigator of this initiative is Michael W. Weiner, MD, VA Medical Center and University of California-San Francisco. ADNI is the result of efforts of many coinvestigators from a broad range of academic institutions and private corporations, and subjects have been recruited from more than 50 sites across the United States and Canada. The initial goal of ADNI was to recruit 800 subjects, but ADNI has been followed by ADNI-GO and ADNI-2. To date these three protocols have recruited more than 1500 adults, aged 55 to 90 years, to participate in the research, consisting of cognitively normal older individuals, people with early or late MCI, and people with early $\mathrm{AD}$. The follow-up duration of each group is specified in the protocols of ADNI-1, ADNI-2, and ADNI-GO. Subjects originally recruited for ADNI-1 and ADNI-GO had the option to be followed in ADNI-2. For up-to-date information, see www.adni-info.org. In the current study, we used ADNI-1 data downloaded on 17 October 2012.

\subsection{Participants}

ADNI-1 general eligibility criteria are described at www. adni-info.org/Scientists/ADNIGrant/ProtocolSummary.aspx. Healthy controls had a Mini-Mental State Examination (MMSE) score between 24 and 30 (inclusive), a global Clinical Dementia Rating (CDR) [5] score of 0, and did not meet criteria for MCI or dementia [6,7]. MCI participants had MMSE scores between 24 and 30 (inclusive), a memory complaint, evidence of objective memory loss as measured by education adjusted scores on 
CSF T-tau (U2 series) in $\mathrm{pg} / \mathrm{mL}$
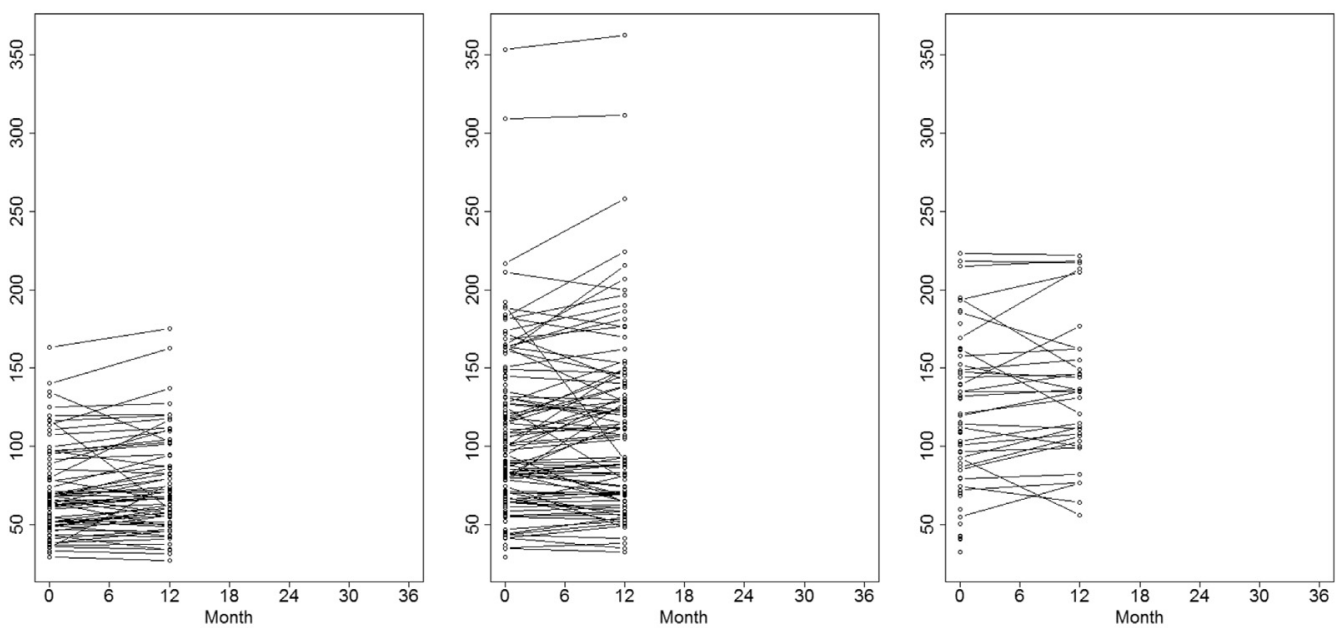

CSF AB42 (U2 series) in $\mathrm{pg} / \mathrm{mL}$
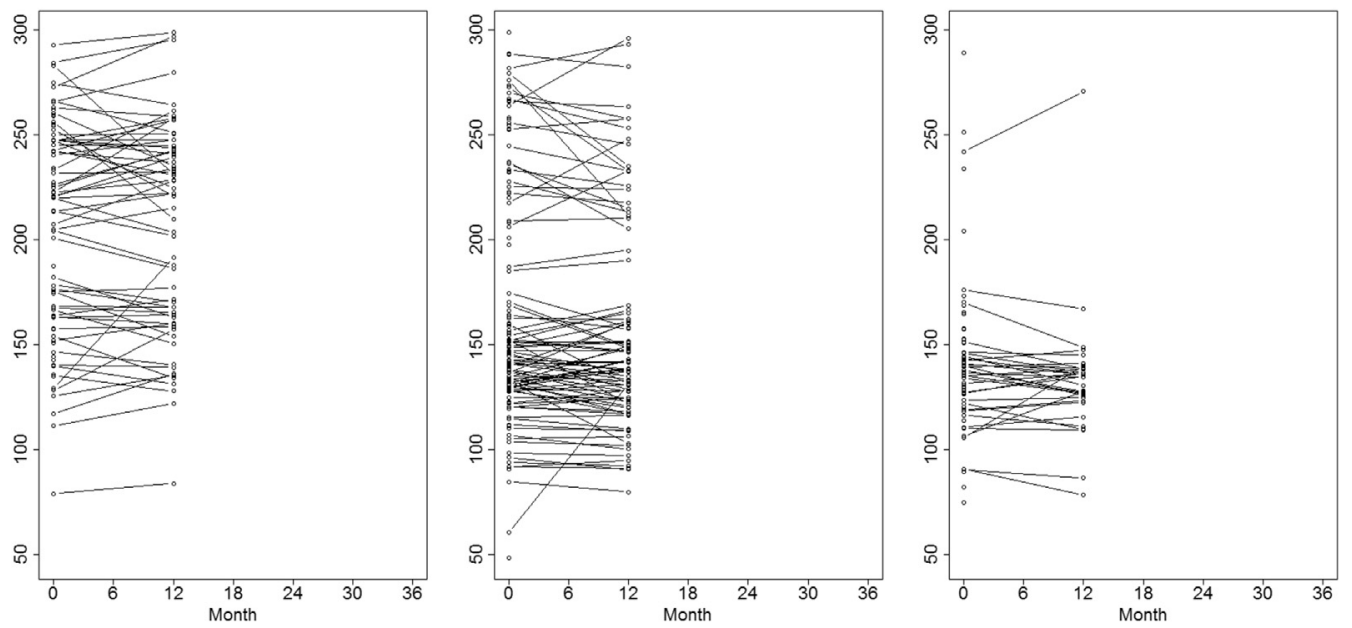

Fig. 1. (continued).

the Wechsler Memory Scale Logical Memory II, a CDR of 0.5 , absence of significant levels of impairment in other cognitive domains, essentially preserved activities of daily living, and absence of dementia. Mildly demented AD participants had MMSE scores between 20 and 26, global CDR scores of 0.5 or 1.0 , and met the National Institute of Neurological and Communicative Disorders and Stroke and the Alzheimer's Disease and Related Disorders Association (NINCDS/ADRDA) criteria for probable AD [8].The ADNI-1 study collected cerebrospinal fluid (CSF) and fluorodeoxyglucose positron emission tomography (FDG-PET) from approximately 50\% of participants at baseline, and from smaller subgroups subsequently. CSF and FDG-PET biomarker acquisition procedures for ADNI are described in detail elsewhere [9-11]. Participants with CSF data, and baseline and follow-up MRI scans (interval data) that met global quality control criteria were used in the current analysis (see http://adni.loni.usc.edu/ wp-content/uploads/2010/09/ADNI_MRI_Tech_Proc_Ma nual.pdf). Baseline and slopes of progression of the following biomarkers and their association with declines in cognitive function (described later) were examined: MRI total brain volumes, hippocampal volumes, ventricular volumes, white matter hyperintensity (WMH) volumes, CSF total tau protein (t-tau) and $A \beta_{42}$ levels, cortical thickness of selected regions (precuneus and medial temporal cortical thickness, the latter being the summary variable obtained by adding averaged means for left and right entorhinal, perirhinal, and posterior parahippocampal cortical region thicknesses [12,13]), and FDG-PET (summary variable by averaged mean for left and right temporal, right, and left angular and posterior cingulate cortices $[9,14])$. Biomarker assessment protocols are described in detail at http://adni.loni.usc.edu/wp-content/ uploads/2010/09/CSF_Biomarker_Test_Instr.pdf. A total of 526 subjects (156 with normal cognition, 262 with MCI and 108 with AD using baseline diagnosis) with valid data for our variables of interest were used in this study. By study design, sample size differs depending on biomarkers. 

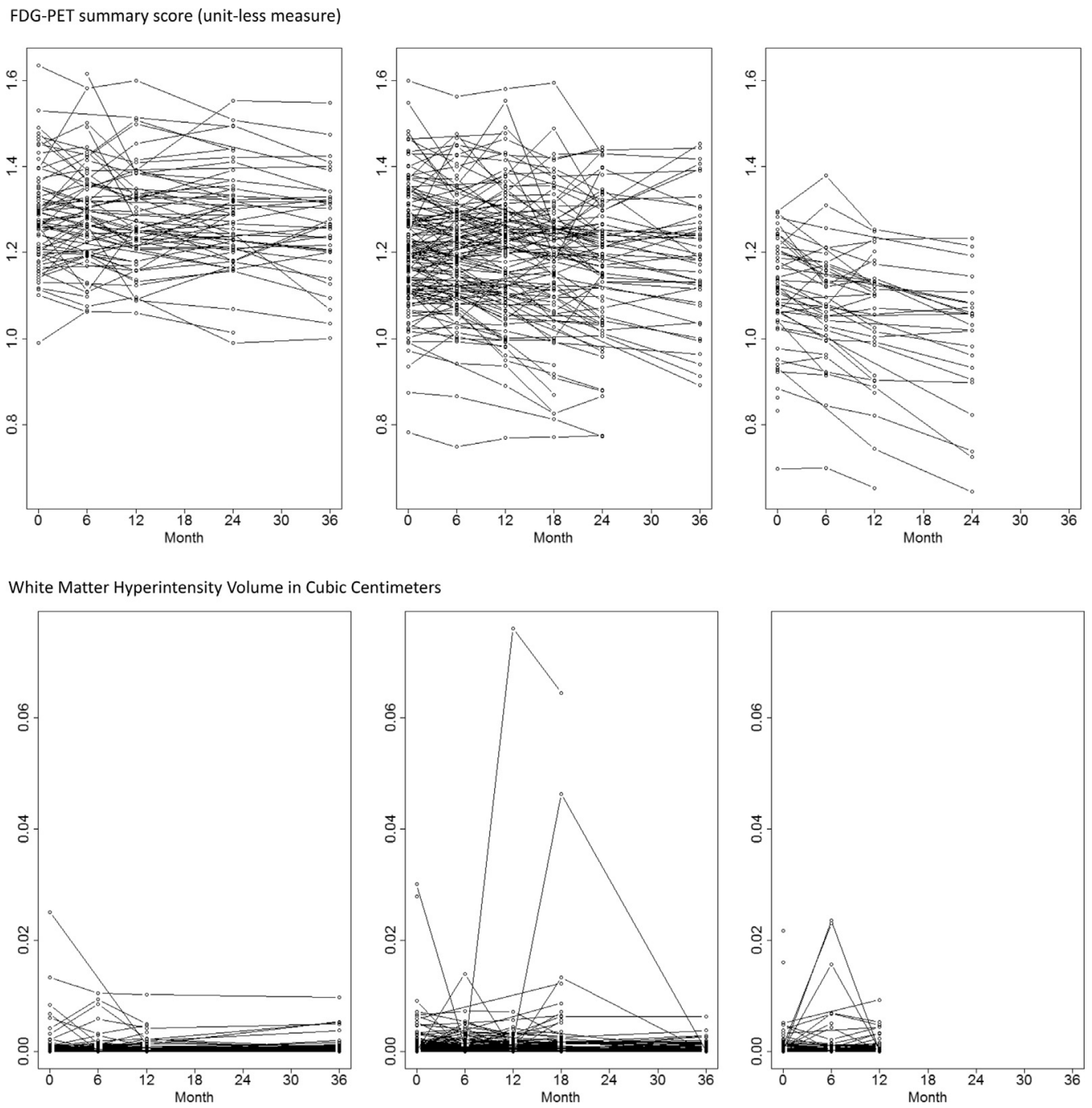

Fig. 1. (continued).

\subsection{Cognitive outcomes}

Trajectories (age slope) of cognitive domain measurements examined are ADNI-memory (ADNI-Mem) and ADNIexecutive function (ADNI-Exe) $[15,16]$.The scores are psychometrically optimized composite scores of memory and executive function, respectively, derived from items from ADNI NP tests. These measurements were validated previously, robust, and have external validity [15-17].

\subsection{Statistical analyses}

Individual-specific slopes (i.e., random component) of the longitudinal trajectory of each biomarker were estimated using mixed effects models. We defined changes in diagnosis when subjects remained in a new diagnostic category for at least two consecutive assessments. Less than three cases changed diagnosis among the control group using this criterion. Therefore, we did not include the indicator variable for these subjects when analyzing control subjects. These estimates and observed baseline values were used as predictors of cognitive decline (decline in ADNI-Mem and
ADNI-Exe) using mixed effects models, controlling for age, sex, Apolipoprotein E (APOE) $\varepsilon 4$ allele status ( $\varepsilon 4$ present or absent), years of education, and practice effects. Practice effects, which refer to improvements in cognitive test scores over repeated administrations, are often observed at the second and third assessments, especially among those with normal cognition or early stage MCI $[18,19]$. The magnitude of practice effects may be an indicator of future cognitive decline, but the normative magnitude of practice effects and precise timing of when practice effects subside are not established. Therefore, we first examined the amount and timing of practice effects by assessing changes in cognitive slopes using a piecewise approach (i.e., allowing slope changes between baseline and sixth-month assessment, between sixth month assessment and year 1 assessment, etc.). This change point analysis identified that for memory function (ADNI-Mem), model fitness is best if we assume that practice effect peaked at the sixth month assessment, and for executive function (ADNI-Exe) at the 12th month assessment. We evaluated indicators allowing shifts in slopes beyond 12th month assessment, but they 

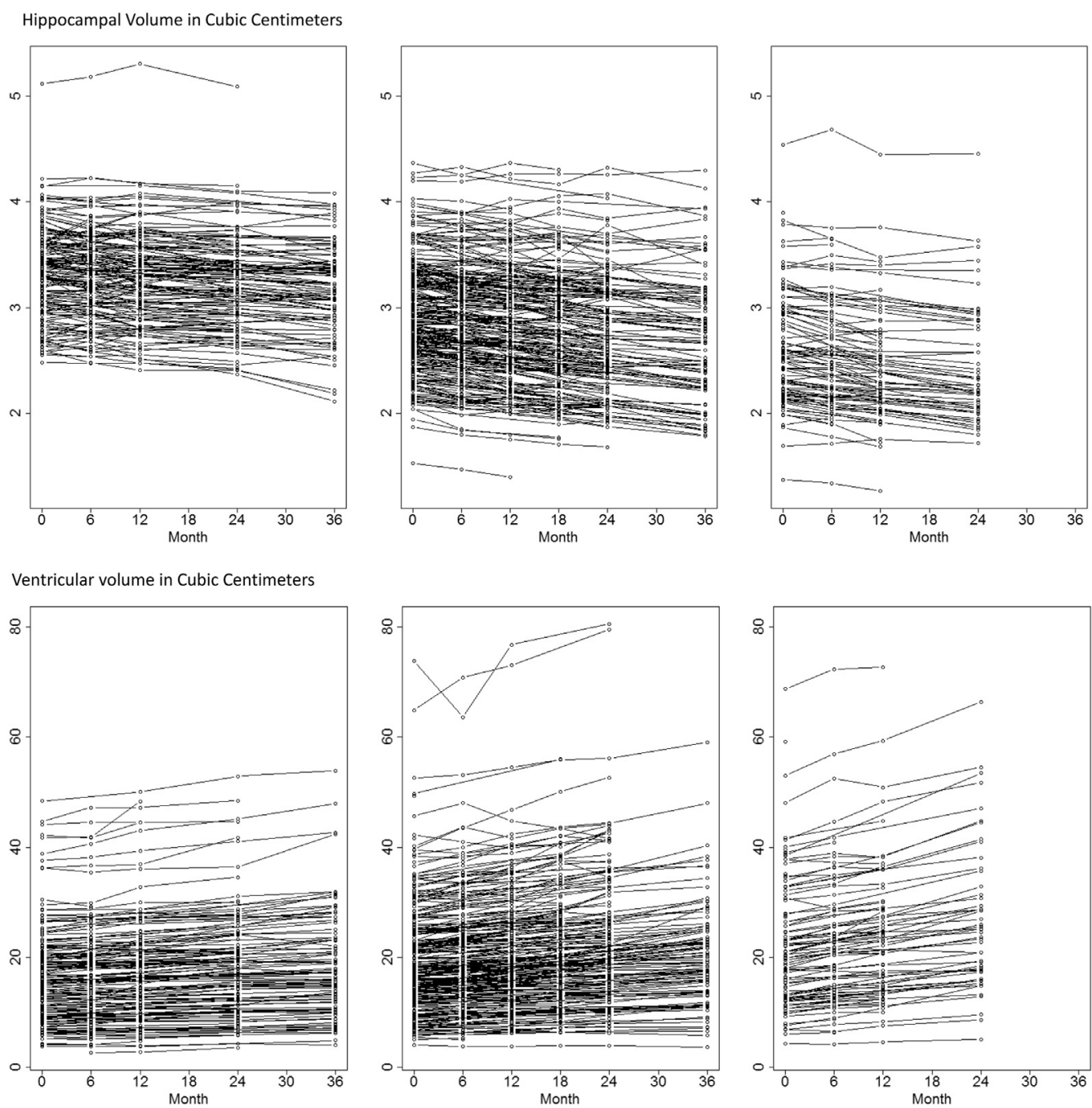

Fig. 1. (continued).

were not significant. That is, the change point in slope occurs only at sixth month assessment (for memory) and at year 1 (for executive function) and cognitive function declines continuously thereafter. Accordingly, this change point was included in the model to control for practice effects (see Appendix). Variability in cognitive decline (i.e., individual differences in slopes) explained by the subject-specific baseline biomarker values was compared with variability in cognitive decline explained by subject-specific biomarker progressions. Each model included time in months $(0,6$, $12,18,24$, and 36 months from baseline). Baseline biomarker values and time interactions, and biomarker progressions and time interactions were added separately to examine the variability in random slopes in cognitive declines explained by each component (baseline biomarker values vs. biomarker progressions). Each brain volume was divided by intracranial volume (ICV). All biomarker variables were standardized so that estimated effects of biomarkers on cognitive decline could be meaningfully compared across different biomarkers. WMH/ICV was normalized first with a log transformation due to its skewed distribution. All biomarkers were standardized using baseline means and standard deviations. Intercept and time were treated as random effects in all models. All models assumed an unstructured withinsubject error covariance structure and restricted maximum likelihood was used for estimation. We applied the same mixed effects models to each stage separately (controls, $\mathrm{MCI}$, and $\mathrm{AD}$ ) to examine the predictive effect of biomarkers on cognitive declines within each group. A variable indicating changes in diagnosis (from MCI to AD) was included when analyzing subjects in MCI group. The overall fit of the models was examined using a combination of formal fit criteria and visual inspection of residual plots. Results were considered significant at $P<.05$.

\section{Results}

\subsection{Characteristics of study samples}

Table 1 shows the baseline characteristics of study samples by diagnostic category at baseline. As shown, CSF, t-tau, and A $\beta 42$ (U2 series) were assessed only 

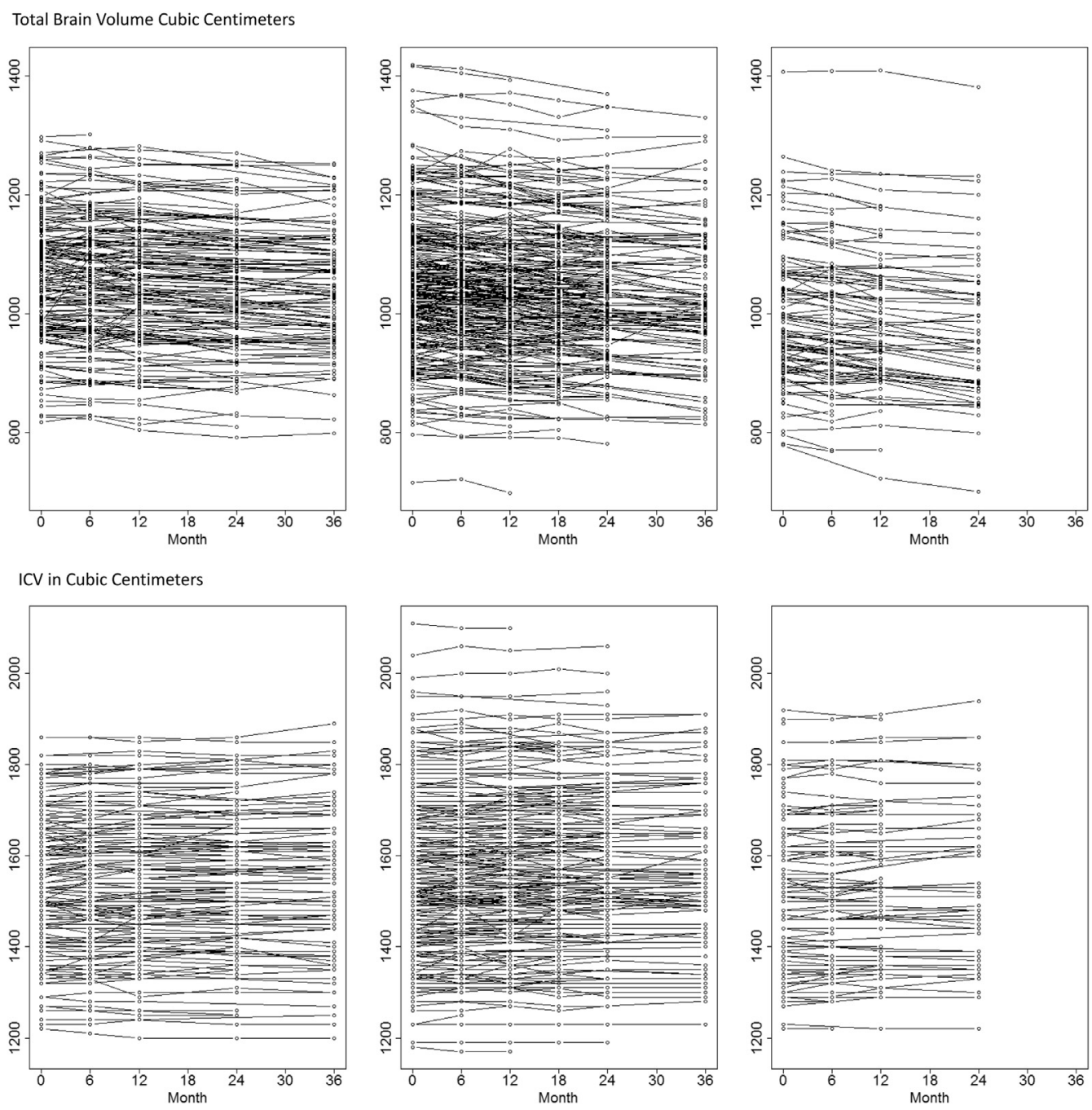

Fig. 1. (continued).

up to twice in ADNI-1. Figure 1shows spaghetti plots of cognitive functions and biomarkers used in the analysis.

\subsection{Cognitive declines}

Table 2 shows the proportion of variability in ADNI-Mem and ADNI-Exe declines over time explained by baseline biomarker values or biomarker progressions. Table 2 is read as follows: Among MCI subjects, for example, one standard deviation larger expansion in ICV-adjusted ventricular volume is associated with 0.12 further decline in memory scores each year (slope effect: -0.12 ) and biomarker progression explains $39.4 \%$ of variability in cognitive decline, whereas the baseline value explains only $8.7 \%$ of variability in memory decline. When inclusion of biomarker baseline and progression values in the model causes more estimation errors (i.e., noise, indicated by increased variability in ADNI-Mem and ADNI-Exe declines), we noted N/A in Table 2.

\subsubsection{Memory decline}

Among normal subjects, FDG-PET score biomarker progression, precuneus thickness baseline and progression, and medial temporal lobe thickness progression explained variability in memory declines, but only to a limited extent: $1.5 \%, 4.5 \%, 1.2 \%$, and $4.4 \%$, respectively. Among MCI subjects, several biomarkers predicted memory decline; biomarkers which explained the most variability were progression of ventricular volumes $(39.4 \%)$, followed by shrinkage of medial temporal cortical thickness (28.7\%), and hippocampal atrophy (19.8\%). For all biomarkers except WMH volume and t-tau, biomarker progressions explained a greater fraction of memory decline than baseline values. Differences between baseline and progression in explaining variability of memory declines were especially large for total and ventricular brain volume atrophy, with five times or more variability explained by biomarker progressions than baseline values. Among AD subjects, much higher proportions of variability were explained by biomarker progressions than by baseline biomarker values. 

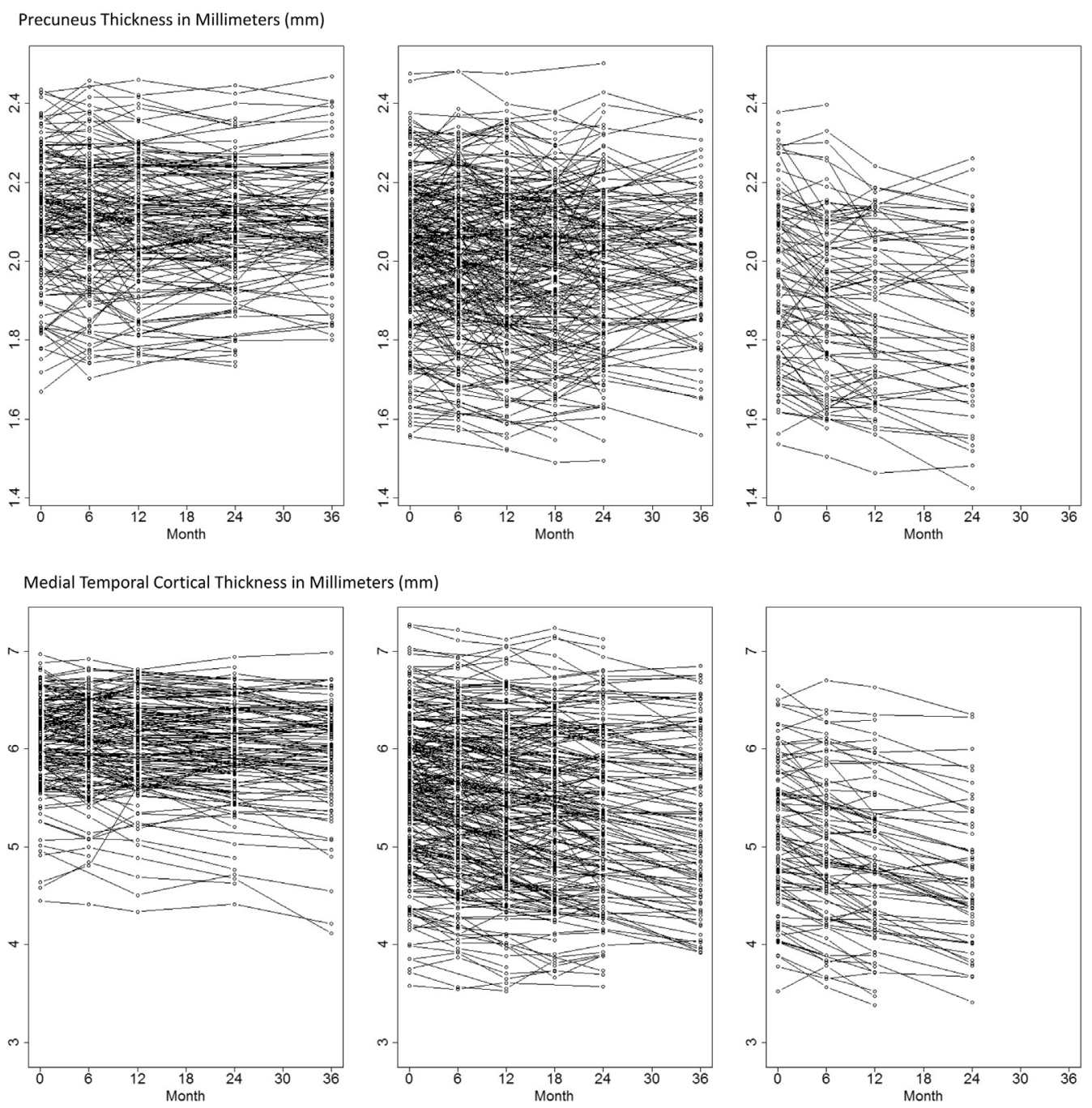

Fig. 1. (continued).

Differences in explanatory capacities between baseline values and biomarker progressions were larger than those observed among MCI subjects. The highest proportion of variability in ADNI-Mem changes was explained by progression of FDG-PET scores (84\%), followed by ventricular volume loss $(63.8 \%)$. Looking at each biomarker across diagnosis groups, with the exception of CSF A $\beta_{42}$ and $\mathrm{t}$-tau, the explanatory ability of biomarker progression values increased with progression from normal through MCI to AD. Progression of CSF A $\beta 42$ level was associated with memory decline more strongly during the MCI stage, although the proportion is not high compared with other biomarkers. The ability of t-tau to predict memory declines was either null or added further variability in ADNI-Mem scores in all diagnostic groups.

\subsubsection{Executive function decline}

Among normal subjects, precuneus thickness (21.1\%) and hippocampal volume $(9.2 \%)$ are the only two biomarkers whose baseline values showed association with executive function declines among normal subjects. CSF A $\beta 42$ progression, FDG-PET progression, ventricular volume progression, and precuneus thickness progression explained the variability in executive functions to some extent $(2.50 \%-$ 9.78\%). Among MCI subjects, as with memory declines, biomarker progressions had stronger associations with executive declines than baseline values. Ventricular volume progression explained the highest variability of executive declines (44.5\%). Unexpectedly among MCI subjects, CSF A 342 explained more variability in executive declines than in memory declines: its baseline value explained $23.7 \%$ and its progression explained $14.4 \%$ of variability in executive declines, whereas the corresponding figures were $5.1 \%$ and $10.3 \%$ in memory declines. Among AD subjects, ventricular volume progression also explained the highest variability of executive function declines $(65.1 \%)$, followed by FDT-PET scores progression (39.5\%). As expected, neither hippocampal volume baseline nor progression explained the variability of ADNI-Exe decline among AD subjects, whereas they did for ADNI-Mem. 
Table 2

Proportion of decline in memory function (ADNI-Mem) explained by each biomarker: baseline and change values. 2A: Memory function (ADNI-Mem) as an outcome. 2B: Executive function (ADNI-Exe) as an outcome

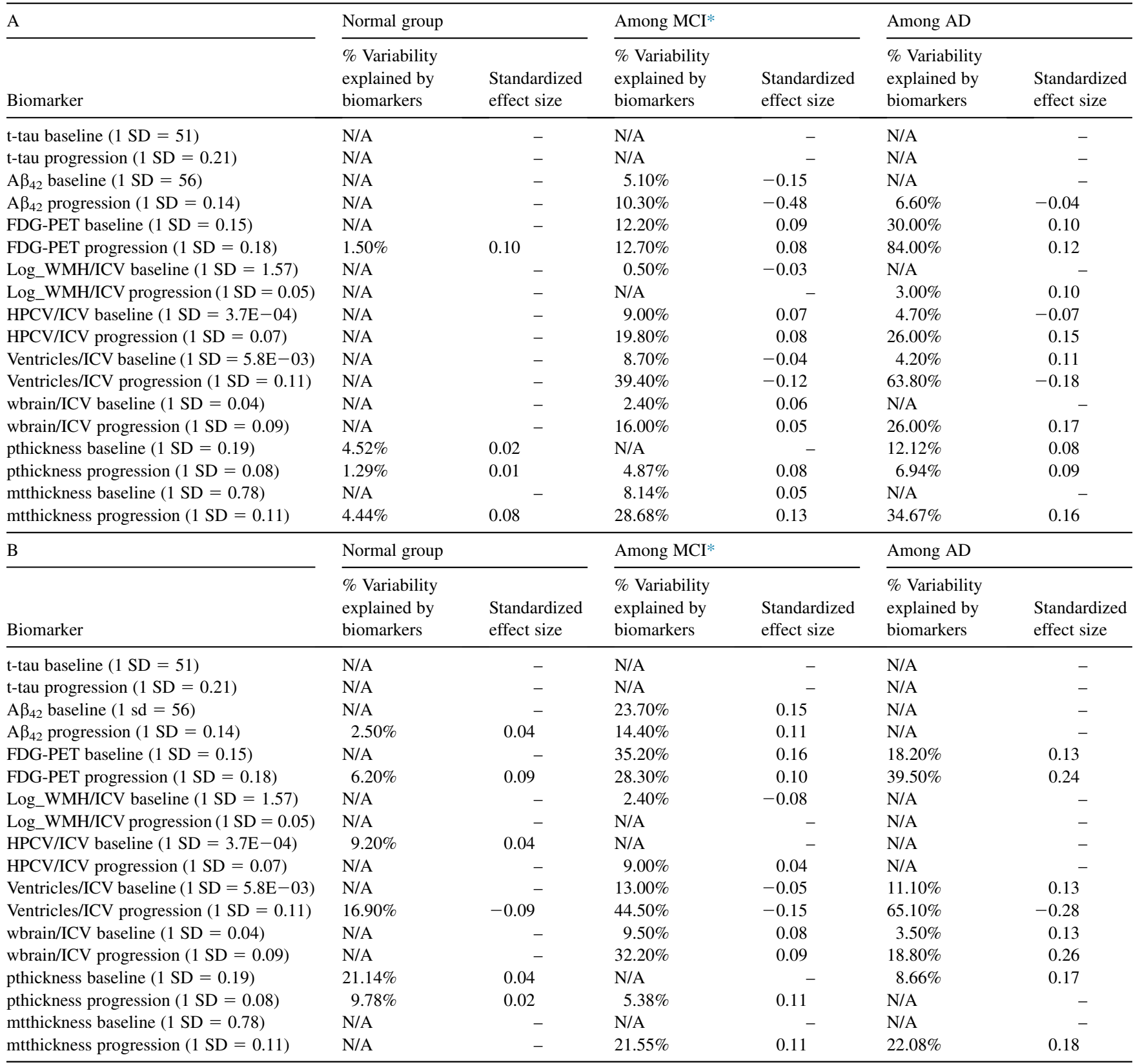

Abbreviations: A $\beta$, amyloid beta; AD, Alzhiemer's disease; ADNI, Alzheimer's Disease Neuroimaging Initiative; FDG-PET, fluorodeoxyglucose positron emission tomography; HPCV, hippocampal volume; ICV, intracranial volume; SD, standard deviation; WMH, white matter hyperintensity; wbrain, total brain volume; pthickness, precuneus thickness; mtthickness, medial temporal cortical thickness.

NOTE. Brain volumes were divided by ICV. Controlling for age at baseline, sex, education, APOE $\varepsilon 4$ allele (at least one vs. none), and practice effects.

N/A: Variability increased instead of decreased or had no changes, after inclusion of the predictors in the model. That is, including these variables did not explain the variability of cognitive outcomes or caused more estimation errors instead of explaining the variability. For these models, goodness of fit of the model compared with the null model did not improve based on $-2 \log$ likelihood ratio tests.

The table can be read as follows: For example, among MCI subjects, one standard deviation larger expansion in ICV-adjusted ventricular volume (ventricles/ ICV progression) is associated with 0.12 further decline in memory scores each year (slope effect: -0.12 , one SD corresponds to $11 \%$ in terms of ventricles/ICV progression) and the progression explains $39.4 \%$ of variability in cognitive decline, whereas baseline value explains only $8.7 \%$ of variability in cognitive decline.

*To capture changes in diagnosis from MCI to AD during the follow-up, an indicator variable (before AD coded as 0, after AD coded as 1) was included as a control variable to factor in the shift in slopes in cognitive decline among MCI. 


\section{Discussion}

Using ADNI-1 data, we examined which components of commonly used AD biomarkers-baseline values or biomarker progressions-explain the variability of declines in memory and executive functions in normal, $\mathrm{MCI}$, and $\mathrm{AD}$ subjects. For most biomarkers, biomarker progressions explained more variability of cognitive declines than baseline values.

A number of studies show support for the hypothetical AD progression model developed by Jack et al. [1,2]. Our study results coincide with the model to some extent; across diagnostic groups, the percentage of variability in cognitive declines explained by functional (FDG-PET) or structural (brain morphometric) biomarkers, either their baseline values or progressions, increased significantly as disease progresses from normal to $\mathrm{AD}$, although structural changes seem to be more closely to or coincided with FDG-PET change than hypothesized [20]. These results reflect that these functional or structural biomarkers change relatively late in a long disease process. This trend was more apparent in memory declines than executive functional declines. Among normal subjects, there was relatively little association between biomarkers and memory decline, but there were more associations between biomarkers and executive decline. This result might be due to the fact that memory decline is minimal among normal subjects and the variability in memory functions does not increase until during later disease stages, whereas executive function declines earlier as a part of normal aging. Among normal subjects, the estimated time slope for memory was -0.01 whereas that for executive functions was -0.04 , suggesting that executive function (ADNI-Exe scores) declined more than memory function (ADNI-Mem). This difference in the magnitude of decline between executive and memory functions among normal subjects could also explain the unexpected result among normal subjects; both CSF A 342 baseline and progression were not associated with memory decline, but CSF A $\beta 42$ progression did explain some of the variation in executive function declines. Also CSF $\mathrm{A} \beta 42$ progression might be related to executive function in MCI because it detects a subgroup of MCI patients with a progressive disorder, not so much because the degree of declines is related to the amount of CSF A $\beta 42$ progression.

Using $\left[{ }^{18}\right.$ F]Florbetapir PET, Landau et al. [21] found that A $\beta$ deposition estimated by $\left[{ }^{18} \mathrm{~F}\right]$ Florbetapir PET was associated with cognitive decline measured by ADAS-Cog retrospectively, but FDG-PET summary score was not, among normal subjects. In our study, FDG-PET progression, precuneus thickness, and medial temporal cortical thickness progressions explained variability of ADNI-Mem score changes across all diagnostic groups, although its predictive ability in the normal group was limited. Similarly, FDG-PET and ventricular volumes progressions were the only biomarkers to explain variability in ADNI-Exe score changes across all diagnostic groups. The outcome measures in our current study are psychometrically optimized distinct indicators of memory and executive functions, whereas the global ADAS-Cog scores combined all cognitive domains. The difference in outcome measures may explain differences in results between those reported by Landau et al. and our current study. Prospective studies are warranted as more data on amyloid PET imaging accumulates.

Looking across the two major cognitive domains examined in this study, memory and executive functions, there was some overlap in individual biomarker progressions' capabilities to explain cognitive declines. In $\mathrm{MCI}$ and AD groups, FDG-PET score changes, ventricular volume increases, whole brain volume declines, and medial temporal cortical thinning progressions generally exhibited parallel and often robust effects in explaining both ADNIMem and ADNI-Exe score declines. Robust association with cognitive change in both domains suggests that these biomarker progressions may be particularly useful in trial design and subject selection. As the field moves toward trials in presymptomatic populations, defining useful biomarkers predictive of cognitive declines during the presymptomatic period is particularly important. Among normal subjects in this data set, precuneus thickness baseline values and medial temporal cortical thinning progressions explained variability in ADNI-Mem declines by more than $4 \%$, showing more explanatory ability than CSF A $\beta 42$. The precuneus is a site of preferential amyloid uptake in $\mathrm{PiB}$ studies, and its thinning is documented as an early sign of AD pathology [22-25]. Dickerson et al. [13], showed that early stage AD is associated with a large degree of medial temporal cortical thinning, although the volumes of medial temporal cortical regions may decrease with both aging and $\mathrm{AD}$. The relatively poor performance of CSF A $\beta 42$ and t-tau biomarker progression values in explaining the cognitive trajectories could be due to shorter duration of follow-up of these markers in comparison with other markers in the current study. Alternatively, the control group in ADNI is likely to be heterogeneous and include significant numbers of individuals who will not progress to $\mathrm{AD}$, although the group has a higher proportion of those with $A P O E$ \& 4 allele than most population norms. Because trials in $\mathrm{AD}$ are increasingly focusing on prevention in asymptomatic individuals, there is a need for markers more strongly associated with cognitive decline during the presymptomatic stage. Further follow-up of normal subjects is required to compare associations of biomarkers and cognitive functions between those who transited to $\mathrm{AD}$ and remained normal.

In most biomarkers, progressions were more strongly associated with cognitive declines measured by NP tests. This suggests that clinical trials recruiting at risk subjects may be improved by using progression rather than baseline values in biomarkers to appropriately enrich study cohorts. Use of biomarker progressions requires at least two assessments before the trial enrollment. Two or three assessments are also required to phase out practice effects in cognitive 
test scores and obtain robust estimates of cognitive declines. Grill et al. [26], estimated required sample sizes per arm for a 36-month trial in detecting differences in changes in cognitive test outcomes using ADNI baseline biomarker information among those with normal cognition. When CDR sum of boxes is an outcome, FDG-PET and lateral ventricle volume values gave relatively small sample sizes; sample sizes required are 1039 given enrichment by FDG-PET summary values, and 1686 given lateral ventricle volume, whereas the required sizes given enrichment by CSF A $\beta$, t-tau, p-tau, and Apoe4 were 1710, 2136, 2901, and 2372, respectively. That is, enriching with FGD-PET baseline scores actually gave the smallest sample size estimates when outcome was CDR-sum of boxes. Future studies are warranted to estimate the incremental effectiveness of improving clinical trial statistical power by using biomarker progression criteria.

WMH expansion was shown to be an early marker associated with MCI incidence [27], and is associated with cognitive decline among nondemented subjects [28-34]. WMH progression was found to be a better predictor of cognitive decline than baseline values [35]. In the current study, WMH did not explain the variability in either memory decline or executive function decline, but rather increased the noise in estimating cognitive declines among normal subjects. The association between WMH expansion and cognitive declines is complex and may be affected by the specific cognitive functions measured and floor effects during disease progression. It is possible also that different regional WMH has differential associations with specific cognitive function declines [35-37]. Alternatively, subjects in ADNI have lower vascular burden due to the study exclusion criteria and the WMH volume is low at enrollment, which may make it difficult to observe associations with cognitive functions. Finally, large measurement variability associated with WMH assessment could lead to our study result. Fluctuation in longitudinal WMH measurement is large. Over a long run with several follow-up assessments, WMH trajectory estimates would become more robust, but when only a few data points are available, estimation of WMH trajectories are vulnerable to measurement fluctuations. Analysis involving WMH (either as outcome or predictor variables) could require more data points than some of other biomarkers examined to obtain robust estimates.

There are several potential limitations of this study. As with other studies using ADNI data, subjects in each diagnostic group were recruited separately, but not followed throughout the disease spectrum. As such, our interpretation of the cascade of pathologic events discussed in this study is limited to inferences. In our preliminary analysis, we confirmed that missingness of biomarkers in ADNI data is not missing completely at randomand is assumed to be missing at random. However, it is possible that missingness is due to informative dropout. Sensitivity analysis can be conducted if more knowledge is available for the informative dropout mechanism.
In conclusion, for most biomarkers, biomarker progressions explained higher variability in cognitive decline than biomarker baseline values. Enriching the recruitment of at-risk subjects using biomarker progressions could improve clinical trials. The incremental effectiveness of improving clinical trial statistical power by using biomarker progression criteria is warranted.

\section{Acknowledgments}

Data collection and sharing for this project were funded by the Alzheimer's Disease Neuroimaging Initiative (ADNI) (National Institutes of Health Grant U01 AG024904). ADNI is funded by the National Institute on Aging, the National Institute of Biomedical Imaging and Bioengineering, and through generous contributions from Alzheimer's Association; Alzheimer's Drug Discovery Foundation; BioClinica, Inc.; Biogen Idec Inc.; Bristol-Myers Squibb Company; Eisai Inc.; Elan Pharmaceuticals, Inc.; Eli Lilly and Company; F. Hoffmann-La Roche Ltd and its affiliated company Genentech, Inc.; GE Healthcare; Innogenetics, N.V.; IXICO Ltd.; Janssen Alzheimer Immunotherapy Research \& Development, LLC.; Johnson \& Johnson Pharmaceutical Research \& Development LLC.; Medpace, Inc.; Merck \& Co., Inc.; Meso Scale Diagnostics, LLC.; NeuroRx Research; Novartis Pharmaceuticals Corporation; Pfizer Inc.; Piramal Imaging; Servier; Synarc Inc.; and Takeda Pharmaceutical Company. The Canadian Institutes of Health Research is providing funds to support ADNI clinical sites in Canada. Private sector contributions are facilitated by the Foundation for the National Institutes of Health (www.fnih.org). The grantee organization is the Northern California Institute for Research and Education, and the study is coordinated by the Alzheimer's Disease Cooperative Study at the University of California, San Diego. ADNI data are disseminated by the Laboratory for Neuro Imaging at the University of California, Los Angeles. This research was also supported by National Institutes of Health grant (P30 AG010129). Additional funding includes National Institute on Aging (P30 AG008017, 1R01AG036772, and R13 AG030995) and Michigan Alzheimer's Disease Center Pilot Grant.

\section{Appendix}

Two-Stage Statistical Models

\section{Model Description}

\section{Stage 1: Longitudinal trajectories of biomarkers}

For any adjusted and standardized biomarker, let $b_{i t}$ denote its value of the $i$ th participant at time $t$, and we assume a linear mixed model for each biomarker:

$$
\begin{gathered}
b_{i t}=\beta_{00}+\beta_{0 i}+\beta_{10} t+\beta_{1 i} t+\gamma_{i t \prime} \\
\text { where }\left(\beta_{0 i}, \beta_{1 i}\right)^{T} \stackrel{i i d}{\sim} \operatorname{MVN}\left(0, \Sigma_{b}\right) \text { and } \gamma_{i t} \stackrel{i i d}{\sim} N\left(0, \sigma_{b}^{2}\right) .
\end{gathered}
$$


The model is fitted in each baseline diagnosis group (normal, MCI, and AD) separately. Estimated subject-specific progression of the biomarker $\widehat{\beta}_{10}+\widehat{\beta}_{1 i}$ is calculated for each subject, where $\widehat{\beta}_{1 i}$ is the subject-specific deviation from $\widehat{\beta}_{10}$, the group average progression of the biomarker. To make the biomarker progression comparable with the baseline values and also comparable across all biomarkers, we pool the subject-specific biomarker progression estimates from three groups together to obtain standardized biomarker progression $\widehat{\beta}_{1 i}^{S}$ with mean 0 and standard deviation 1 .

\section{Stage 2: Association between biomarker baselinel} progression and outcome

We use memory score in mild cognitive impairment group as an example, and similar models are used for memory score and executive function score in all groups. For memory score, model fitting statistics from model diagnostics indicate that slope changes at 6 months $(\mathrm{t}=0.5$ year $)$. Let $y_{i t}$ denote the memory score of the $i$ th participant at time $t$, and we assume the following linear mixed model after model diagnostics:

$$
y_{i t}=I_{i}+S_{10} *\left[1_{\{t<0.5\}} * t\right]+S_{2 i} *\left[1_{\{t \geq 0.5\}} *(t-0.5)\right]+\varepsilon_{i t} ;
$$

$$
\begin{aligned}
I_{t}=\alpha_{0} & +\alpha_{1} * \text { Age }+\alpha_{2} * \text { Education }+\alpha_{3} * \text { Gender }+\alpha_{4} * \text { Apoe } 4 \\
& +\alpha_{5} * b_{i 0}+\alpha_{0 i} ;
\end{aligned}
$$

$$
\begin{aligned}
S_{2 i}=s_{20} & +s_{21} * \text { Gender }+s_{22} * \text { Apoe } 4+s_{23} * \text { Progress to AD } \\
& +s_{24} * b_{i 0}+s_{25} * \widehat{\beta}_{1 i}^{S}+\tau_{2 i} ;
\end{aligned}
$$

where $\varepsilon_{i t} \stackrel{i i d}{\sim} N\left(0, \sigma_{M}^{2}\right)$ and

$$
\left(\alpha_{0 i}, \tau_{2 i}\right)^{T} \underset{i i d}{\sim} \operatorname{MVN}\left(0,\left(\begin{array}{cc}
\sigma_{I}^{2} & \sigma_{I S} \\
\sigma_{I S} & \sigma_{S}^{2}
\end{array}\right)\right)
$$

Because baseline biomarker $b_{i 0}$ and biomarker progression $\widehat{\beta}_{1 i}^{S}$ are standardized across groups, we compare the standardized effects $s_{24}$ and $s_{25}$ for all biomarkers.

Note:

1. For memory score and executive function score in normal and $\mathrm{AD}$ groups, indicator "progress to AD" does not apply and therefore is not included in the level 2 model $S_{2 i}$;

2. For executive function score in all groups, model fitting statistics indicate that slope changes at the third visit (12 months or 1 year), therefore the level one model becomes $y_{i t}=I_{i}+S_{10} *\left[1_{\{t<1\}} * t\right]+S_{2 i} *\left[1_{\{t \geq 1\}} *(t-1)\right]+\varepsilon_{i t}$.

Percentage of variation in outcome progression explained by baseline biomarker and biomarker progression.

For each outcome, we are interested in comparing how much variation in outcome progression is explained by each baseline biomarker and biomarker progression, respectively. To do so, we obtain the estimated variance of the random slope from the following three models, which are the same as the final model described in previous section except for the level 2 slope model $S_{2 i}$ :

Null model: $S_{2 i}$ does not include $b_{i 0}$ or $\widehat{\beta}_{1 i}^{S}$, and $\widehat{\sigma}_{S 0}^{2}$ is the estimated variance of $\tau_{2 i}$;

Baseline-only model: $S_{2 i}$ includes $b_{i 0}$ but not $\widehat{\beta}_{1 i}^{S}$, and $\widehat{\sigma}_{S B}^{2}$ is the estimated variance of $\tau_{2 i}$;

Progression-only model: $S_{2 i}$ includes $\widehat{\beta}_{1 i}^{S}$ but not $b_{i 0}$, and $\widehat{\sigma}_{S S}^{2}$ is the estimated variance of $\tau_{2 i}$.

We then calculate the percentage of variation in slope explained by baseline biomarker and biomarker progression as $\left(\widehat{\sigma}_{S 0}^{2}-\widehat{\sigma}_{S B}^{2}\right) / \widehat{\sigma}_{S 0}^{2} * 100 \%$ and $\left(\widehat{\sigma}_{S 0}^{2}-\widehat{\sigma}_{S S}^{2}\right) / \widehat{\sigma}_{S 0}^{2} * 100 \%$. A positive percentage indicates that the corresponding predictor explains the variation in outcome progression, whereas a negative percentage indicates that inclusion of the predictor adds more estimation error instead of improving model fitting.

\section{RESEARCH IN CONTEXT}

1. Systematic review: We reviewed available English language literature in PubMed up to March 2013 using the search term "Alzheimer's Disease Neuroimaging Initiative" or "ADNI" to find studies that examined predictors of cognitive declines at each stage of Alzheimer disease (AD).

2. Interpretation: In most biomarkers, progressions were more strongly associated with cognitive declines than baseline values. Across diagnostic groups, the percentage of variability in cognitive declines explained by functional or structural biomarkers increased significantly as disease progresses from normal to $\mathrm{AD}$. Among normal subjects, fluorodeoxyglucose positron emission tomography (FDG-PET) score, precuneus, and medial temporal cortical thickness progression and precuneus baseline values explained variability in memory declines, but only to a limited extent.

3. Future directions: (1) Further follow-up of normal subjects is required so that we can compare associations of biomarkers and cognitive functions between those who transited to $\mathrm{AD}$ and remained normal, and identify markers more strongly associated with cognitive decline during the presymptomatic stage. (2) Future studies are warranted to estimate the incremental effectiveness of improving clinical trial statistical power by using biomarker progression criteria. 


\section{References}

[1] Jack CR Jr, Knopman DS, Jagust WJ, Petersen RC, Weiner MW, Aisen PS, et al. Tracking pathophysiological processes in Alzheimer's disease: an updated hypothetical model of dynamic biomarkers. Lancet Neurol 2013;12:207-16.

[2] Jack CR, Knopman DS, Jagust WJ, Shaw LM, Aisen PS, Weiner MW, et al. Hypothetical model of dynamic biomarkers of the Alzheimer's pathological cascade. Lancet Neurol 2010;9:119-28.

[3] Villemagne VL, Burnham S, Bourgeat P, et al. Amyloid beta deposition, neurodegeneration, and cognitive decline in sporadic Alzheimer's disease: a prospective cohort study. Lancet Neurol 2013;12:357-67.

[4] Stricker NH, Dodge HH, Dowling NM, Han SD, Erosheva EA, Jagust WJ. CSF biomarker associations with change in hippocampal volume and precuneus thickness: implications for the Alzheimer's pathological cascade. Brain Imaging Behav 2012; 6:599-609.

[5] Morris JC. The Clinical Dementia Rating (CDR): current version and scoring rules. Neurology 1993;43:2412-4.

[6] Petersen RC, Smith GE, Waring SC, Ivnik RJ, Tangalos EG, Kokmen E. Mild cognitive impairment: clinical characterization and outcome. Arch Neurol 1999;56:303-8.

[7] Petersen RC, Morris JC. Mild cognitive impairment as a clinical entity and treatment target. Arch Neurol 2005;62:1160-3.

[8] McKhann G, Drachman D, Folstein M, Katzman R, Price D, Stadlan EM. Clinical diagnosis of Alzheimer's disease: report of the NINCDS-ADRDA Work Group under the auspices of Department of Health and Human Services Task Force on Alzheimer's Disease. Neurology 1984;34:939-44.

[9] Jagust WJ, Bandy D, Chen K, Foster NL, Landau SM, Mathis CA, et al. The Alzheimer's Disease Neuroimaging Initiative positron emission tomography core. Alzheimers Dement 2010;6:221-9.

[10] Trojanowski JQ, Vandeerstichele H, Korecka M, Clark CM, Aisen PS, Petersen RC, et al. Update on the biomarker core of the Alzheimer's Disease Neuroimaging Initiative subjects. Alzheimers Dement 2010; 6:230-8.

[11] Petersen RC, Aisen PS, Beckett LA, Donohue MC, Gamst AC, Harvey DJ, et al. Alzheimer's Disease Neuroimaging Initiative (ADNI): clinical characterization. Neurology 2010;74:201-9.

[12] Desikan RS, Segonne F, Fischl B, Quinn BT, Dickerson BC, Blacker D, et al. An automated labeling system for subdividing the human cerebral cortex on MRI scans into gyral based regions of interest. Neuroimage 2006;31:968-80.

[13] Dickerson BC, Feczko E, Augustinack JC, Pacheco J, Morris JC, Fischl B, et al. Differential effects of aging and Alzheimer's disease on medial temporal lobe cortical thickness and surface area. Neurobiol Aging 2009;30:432-40.

[14] Landau SM, Harvey D, Madison CM, Koeppe RA, Reiman EM, Foster NL, et al. Associations between cognitive, functional, and FDG-PET measures of decline in AD and MCI. Neurobiol Aging 2011;32:1207-18.

[15] Gibbons LE, Carle AC, Mackin RS, Harvey D, Mukherjee S, Insel P, et al. A composite score for executive functioning, validated in Alzheimer's Disease Neuroimaging Initiative (ADNI) participants with baseline mild cognitive impairment. Brain Imaging Behav 2012;6:517-27.

[16] Crane PK, Carle A, Gibbons LE, Insel P, Mackin RS, Gross A, et al. Development and assessment of a composite score for memory in the Alzheimer's Disease Neuroimaging Initiative (ADNI). Brain Imaging Behav 2012;6:502-16.

[17] Habeck C, Risacher S, Lee GJ, Glymour MM, Mormino E, Mukherjee S, et al. Relationship between baseline brain metabolism measured using $\left[{ }^{18}\right.$ F]FDG PET and memory and executive function in prodromal and early Alzheimer's disease. Brain Imaging Behav 2012;6:568-83.
[18] Howieson DB, Carlson NE, Moore MM, Wasserman D, Abendroth CD, Payne-Murphy J, et al. Trajectory of mild cognitive impairment onset. J Int Neuropsychol Soc 2008;14:192-8.

[19] Dodge HH, Wang CN, Chang CC, Ganguli M. Terminal decline and practice effects in older adults without dementia: the MoVIES project. Neurology 2011;77:722-30.

[20] Sperling RA, Aisen PS, Beckett LA, Bennett DA, Craft S, Fagan AM, et al. Toward defining the preclinical stages of Alzheimer's disease: recommendations from the National Institute on Aging-Alzheimer's Association workgroups on diagnostic guidelines for Alzheimer's disease. Alzheimers Dement 2011;7:280-92.

[21] Landau SM, Mintun MA, Joshi AD, Koeppe RA, Petersen RC, Aisen PS, et al. Amyloid deposition, hypometabolism, and longitudinal cognitive decline. Ann Neurol 2012;72:578-86.

[22] Rabinovici GD, Jagust WJ. Amyloid imaging in aging and dementia: testing the amyloid hypothesis in vivo. Behav Neurol 2009; $21: 117-28$.

[23] Dore V, Villemagne VL, Bourgeat P, Fripp J, Acosta O, Chetelat G, et al. Cross-sectional and longitudinal analysis of the relationship between Abeta deposition, cortical thickness, and memory in cognitively unimpaired individuals and in Alzheimer disease. JAMA Neurol 2013;70:903-11.

[24] Becker JA, Hedden T, Carmasin J, Maye J, Rentz DM, Putcha D, et al. Amyloid-beta associated cortical thinning in clinically normal elderly. Ann Neurol 2011;69:1032-42.

[25] Ye BS, Seo SW, Yang JJ, Kim HJ, Kim YJ, Yoon CW, et al. Comparison of cortical thickness in patients with early-stage versus late-stage amnestic mild cognitive impairment. Eur J Neurol 2014; 21:86-92.

[26] Grill JD, Di L, Lu PH, Lee C, Ringman J, Apostolova LG, et al. Estimating sample sizes for predementia Alzheimer's trials based on the Alzheimer's Disease Neuroimaging Initiative. Neurobiol Aging 2013;34:62-72.

[27] Silbert LC, Dodge HH, Perkins LG, Sherbakov L, Lahna D, ErtenLyons D, et al. Trajectory of white matter hyperintensity burden preceding mild cognitive impairment. Neurology 2012;79:741-7.

[28] Mungas D, Harvey D, Reed BR, Jagust WJ, DeCarli C, Beckett L, et al. Longitudinal volumetric MRI change and rate of cognitive decline. Neurology 2005;65:565-71.

[29] van den Heuvel DM, ten Dam VH, de Craen AJ, Admiraal-Behloul F, Olofsen H, Bollen EL, et al. Increase in periventricular white matter hyperintensities parallels decline in mental processing speed in a non-demented elderly population. J Neurol Neurosurg Psychiatry 2006;77:149-53.

[30] Carmichael O, Schwarz C, Drucker D, Fletcher E, Harvey D, Beckett L, et al. Longitudinal changes in white matter disease and cognition in the first year of the Alzheimer disease neuroimaging initiative. Arch Neurol 2010;67:1370-8.

[31] Silbert LC, Nelson C, Howieson DB, Moore MM, Kaye JA. Impact of white matter hyperintensity volume progression on rate of cognitive and motor decline. Neurology 2008;71:108-13.

[32] Kramer JH, Mungas D, Reed BR, Wetzel ME, Burnett MM, Miller BL, et al. Longitudinal MRI and cognitive change in healthy elderly. Neuropsychology 2007;21:412-8.

[33] Longstreth WT Jr, Arnold AM, Beauchamp NJ Jr, Manolio TA, Lefkowitz D, Jungreis $C$, et al. Incidence, manifestations, and predictors of worsening white matter on serial cranial magnetic resonance imaging in the elderly: the Cardiovascular Health Study. Stroke 2005;36:56-61.

[34] van Dijk EJ, Prins ND, Vrooman HA, Hofman A, Koudstaal PJ, Breteler MM. Progression of cerebral small vessel disease in relation to risk factors and cognitive consequences: Rotterdam Scan study. Stroke 2008;39:2712-9.

[35] Silbert LC, Howieson DB, Dodge H, Kaye JA. Cognitive impairment risk: white matter hyperintensity progression matters. Neurology 2009; $73: 120-5$. 
[36] Prins ND, van Dijk EJ, den Heijer T, Vermeer SE, Jolles J, Koudstaal PJ, et al. Cerebral small-vessel disease and decline in information processing speed, executive function and memory. Brain 2005;128:2034-41.
[37] van Straaten EC, Harvey D, Scheltens P, Barkhof F, Petersen RC, Thal LJ, et al. Periventricular white matter hyperintensities increase the likelihood of progression from amnestic mild cognitive impairment to dementia. J Neurol 2008;255:1302-8. 